\title{
Comparative Study of the Interaction of Digital Natives with Mainstream Web Mapping Services $^{\star}$
}

\author{
Marinos Kavouras ${ }^{1}$, Eleni Tomai ${ }^{1}$, Margarita Kokla ${ }^{1}$, Fotis Liarokapis ${ }^{2,3}$, and \\ Katerina Pastra ${ }^{4}$ \\ 1 Cartography Laboratory, National Technical University of Athens, Zografou \\ Campus, 15780 Zografou, Athens, Greece \\ \{mkav, etomai, mkokla\}@mail.ntua.gr \\ http://www.survey.ntua.gr \\ 2 Research Centre on Interactive Media, Smart Systems and Emerging Technologies, \\ Nicosia 1011, Cyprus \\ f.liarokapis@cyens.org.cy \\ 3 Cyprus University of Technology, Faculty of Engineering and Technology, Limassol \\ 3036, Cyprus \\ fotios.liarokapis@cut.ac.cy \\ 4 Institute for Language and Speech Processing, ATHENA Research Center, 15125 \\ Maroussi, Athens, Greece \\ kpastra@athenarc.gr
}

\begin{abstract}
We present the results of a comparative study among four well-known web mapping services. The study explored how digital natives (young people under 30 years old who were born and raised with technology and smart devices) interact with web mapping services. The sample consisted of 167 University students in the field of Engineering and was conducted entirely online. Results indicate that the nature of tasks performed using mapping services affect both effectiveness and efficiency even when users interact with the same service. Among services, study results also indicate differences between task success rates as well as between successful task completion times. Finally, comparison between male and female participants showed no difference in either effectiveness or efficiency between genders.
\end{abstract}

Keywords: Comparative Study - Web Mapping Service · Efficiency · Effectiveness · Digital Natives.

\footnotetext{
* The research work was supported by the Hellenic Foundation for Research and Innovation (H.F.R.I.) under the "First Call for H.F.R.I. Research Projects to support Faculty members and Researchers and the procurement of high-cost research equipment grant" (ProjectNumber: HFRI-FM17-2661). This research was also partially supported by the project that has received funding from the European Union's Horizon 2020 research and innovation programme under grant agreement No 739578 (RISE-Call: H2020-WIDESPREAD-01-2016-2017-TeamingPhase2) and the Government of the Republic of Cyprus through the Directorate General for European Programmes, Coordination and Development.
} 


\section{Introduction}

The current study aims at exploring the ways digital natives [1] interact with web mapping services. Digital natives were selected because they belong to the Millennials; the generation born and raised with technology. Digital Natives are overexposed to information and communications technologies and to the use of smart devices, thus according to Prensky (ibid.), they exhibit special skills and characteristics such as enjoying multitasking, processing information fast and in parallel, feasting on technology and so on. If we are to adopt this view, digital natives present the ideal subjects for evaluating usability of web mapping services in an era of ever-increasing digital applications and environments.

ISO 9241-11 (1998) in [2] defines usability as "the extent to which a system, product or service can be used by specified users to achieve specified goals with effectiveness, efficiency, and satisfaction in a specified context of use". The revised version (ISO 9241-11, 2018) in [3] adds to the term product the terms system and service. By definition, usability is essentially defined as the combination of three components: (a) effectiveness, refers to whether users can complete tasks and accomplish goals using this system, product or service; (b) efficiency, the degree to which users spend resources to achieve their goals and (c) satisfaction, the level of comfort that users feel when achieving their goals [2].

The study presented herein measures two components of usability; efficiency and effectiveness. Effectiveness refers to whether participants correctly completed the tasks they had to perform using the services, while efficiency refers to their speed in performing a task.

The paper is organized as follows: Section 2 presents related work on the usability of web mapping services. It seems that regarding mapping services excessive usability studies have not been conducted, although a vast global population uses them on their smart devices. Section 3 presents the study methodology detailing procedure and the questionnaires given to participants. Section 4 provides information of the sample's demographics and Section 5 presents results of the analysis. Finally, the last section discusses general conclusions.

\section{Related Work}

As more and more users worldwide use web mapping services either through mobile devices or on a personal computer, evaluating the usability of these services is useful and imperative. Indeed, in recent years, efforts have been made to evaluate well-known and widely used cartographic services (e.g., Google Maps, Yahoo Maps, etc.) without, however, being able to talk of large-scale usability evaluation of web mapping services. The following paragraphs present related surveys focusing on the adopted research/ experimental process of defining and measuring usability of web mapping services.

The usability study of [4] aimed at identifying possible usability problems of web mapping services with the objective of collecting quality information and of proposing guidelines for the future design of relevant services. The survey 
evaluated four services: Google Maps, MSN Maps \& Directions (Bing Maps ancestor), MapQuest and Multimap (merged by Bing Maps). To conduct the study, a typical scenario of web mapping services use was presented to participants: "A tourist plans to visit London and uses a web mapping service to plan the trip in advance". A total of 24 participants ( 8 general users, 8 cartographers, and 8 usability engineers) made 32 different assessments. Users were given a predefined task at a time, which they would complete using one of the services under review that they had not used before. Users were encouraged to "think aloud" and to describe the rationale behind their actions. During the endeavors, the user's computer screen was recorded with a video camera to facilitate the analysis that would follow. General users had to perform seven tasks. Expert users, on the other hand, received the scenario and the same list of tasks. After performing the tasks, they were asked to list all the usability problems they identified. Usability problems were grouped into four different categories depending on the severity of the problem.

A usability framework for web mapping services was proposed with four levels of usability [5]: (1) usability typology (includes the content of the service, the functions and the way of interaction as well as the objects through which the interaction is achieved), (2) usability variables (related to the user and the purpose of using the application, the tasks performed through the use of the application, and the skills that users must have in order to use it), usability data (also referred to as usability characteristics/ properties, which are the measurable components of the usability concept), and (4) usability measures (those characteristics that can be quantified and therefore directly measured or observed during a usability test). Applying this framework, the researchers conducted a survey to investigate the relationship between web mapping service and user satisfaction. The selected services selected: Map24.com (operational until 2011 considered the forerunner of the HERE WeGo) and Mappy.com (launched in France in 1997, covering all of Europe) and user interaction was based on windows, icons, menus, pointers allowing the user to navigate and search for information with the mouse or keyboard (Level 1). For Level 2, a pre-test two-fold questionnaire was designed that allowed the collection (a) of user profile information (occupation, age, and gender) and (b) of web mapping services usage information (e.g., user preferences and usage purposes). Participants performed 3 tasks related to finding a route and locating an object. As a usability element (Level 3) in usability research, "user satisfaction" was measured as the combination of three parameters (Level 4): (1) execution speed, (2) degree of interaction, and (3) error rate. The usability survey was conducted by eight participants and their entire interview and interaction with the services was recorded.

The survey on usability of web mapping services conducted by [6] consisted of the evaluation of: Google Maps, Bing maps, MapQuest, and Yahoo Maps. The services were evaluated by 42 people, with different skills of Geographic Information Systems (ordinary users but also experts), regardless of gender, age, and nationality. The sample was divided into groups and performed various tasks using the services. The research consisted of three parts: the pre-test which 
concerned the collection of the sample's basic information (age, gender, level of familiarity with GIS, etc.), the execution of various tasks, and one post-test questionnaire. The scenario of the experiment was as follows: participants were travellers visiting for the first time the US capital, Washington, DC. In this context they had to perform five tasks. The final quantitative results were obtained partly on the basis of the average time for the execution of each task in the four services and the percentage of successful execution of the tasks.

\section{$3 \quad$ Methodology}

\subsection{Study Research Question}

The study consists in evaluating four web mapping services by undergraduate University engineering students constituting an age group that could provide interesting insights on how youth in the digital era explores and interacts with geo-services, as they are the so-called "digital natives". The study design (Section 3.4) allowed exploring also gender differences, if any, between male and female participants in their interaction with web mapping services.

\subsection{Procedure}

The study was undertaken online during the spring semester of the Academic Year 2019-2020. Due to special conditions, related to the COVID19 outbreak, the study was conducted in the framework of distance learning environments. Participants had been connected to the course team on Microsoft Teams and instructions for the study procedure were given to them orally by the research team. They were asked to perform the survey using their own laptops and PCs. The research team was present during the study to assist participants in any problem they faced either with the understanding of the tasks they had to perform or with any internet connection issues they might face and all interactions were made on Microsoft Teams. The study was conducted in a time frame of one hour and half, between 12:30 and 14:00 pm, so that all participants could take part in the study simultaneously. Data was collected and processed anonymously.

\subsection{Pre-test Questionnaire}

The pre-test questionnaire was designed to collect data on sample demographics such as gender, year of birth, familiarity with computers and smart devices etc. Details on sample demographics as derived from this initial questionnaire are given in Section 4.

\subsection{Web Mapping Services Usability Questionnaire}

We evaluated the four web mapping services in pairs, so three versions of the web mapping services questionnaire were designed. All participants evaluated Google 
Maps, since it is the most used mapping service worldwide [7]. The remaining three services are: Bing Maps, MapQuest, and Here WeGo. Therefore, the sample was equally and randomly divided into three groups that each evaluated two services with Google Maps being the benchmark evaluated by all.

The tasks the participants were asked to perform are:

1. identifying a location (point) given its geographical coordinates and

2. locating the nearest point of interest to a specific point.

The students were given the following "scenario": You have been admitted to Harvard University to attend some classes during a summer school. Before you leave, you want to plan your trip and see which places you will visit. This scenario made the survey process more appealing to the students but also Boston as a US city presented a safe choice since our students would have to perform the task in an unfamiliar environment and because the four mapping services are fully functional for this part of the world ensuring that our participants could not face an issue in successfully executing any task asked from them.

For each task, the following data were recorded and analysed:

- the score in executing the task (score 1 for successful execution, 0 otherwise) and

- the response time (time it took for participants to complete the task regardless of whether it was successful or not).

\section{Sample Demographics}

The sample consists of 167 University students, below the age of 30 years old, of which $113(68 \%)$ are males and $54(32 \%)$ females. The mean age of participants is $21.80( \pm 2.06)$ years old.

Participants' degree of familiarity with computers and smart devices is quite high since almost $50 \%$ of the sample responded that they are very familiar and almost $27 \%$ that they are extremely familiar. Only $22 \%$ considered themselves as moderately familiar. Table 1 details participants' answers and percentages regarding familiarity with computers and smart devices by gender as well as for the sample as a whole.

Table 1. Male, female and total participants' answers and percentages to their degree of familiarity with computers and smart devices.

\begin{tabular}{|l|l|l|l|}
\hline Degree of familiarity & Males(\%) & Females(\%) & Total(\%) \\
\hline Slightly familiar & $2(1.8 \%)$ & $0(0.0 \%)$ & $\mathbf{2 ( 1 . 2 0 \% )}$ \\
Moderately familiar & $26(23.0 \%)$ & $11(20.4 \%)$ & $\mathbf{3 7 ( 2 2 . 1 \% )}$ \\
Very familiar & $51(45.1 \%)$ & $32(59.3 \%)$ & $\mathbf{8 3 ( 4 9 . 7 \% )}$ \\
Extremely familiar & $34(30.1 \%)$ & $11(20.4 \%)$ & $\mathbf{4 5 ( 2 7 . 0 \% )}$ \\
\hline
\end{tabular}

Answers to the question that explores the reasons why participants use computers and smart devices (they were enabled to choose multiple answers) are 
depicted on Table 2. Percentages by gender, reveal that education, entertainment and social networking are the three most prevalent reasons for using these media regardless of gender, with similar percentages between men and women. In fact, these percentages in all cases exceed $25 \%$ of the answers, with the highest percentage of them being the answers of female participants related to education $(28.2 \%)$. As expected, the percentage of answers related to work is much lower $(17.8 \%$ of the sample) since the sample consists of active students who the majority of them do not have a regular job yet. Finally, regarding the degree of

Table 2. Male, female and total participants' answers and percentages on the reasons for using computers and smart devices.

\begin{tabular}{|l|l|l|l|}
\hline Reasons of Use & Males(\%) & Females(\%) & Total(\%) \\
\hline Work & $72(18.5 \%)$ & $29(16.4 \%)$ & $\mathbf{1 0 1 ( 1 7 . 8 \% )}$ \\
Education & $107(27.5 \%)$ & $50(28.2 \%)$ & $\mathbf{1 5 7}(\mathbf{2 7 . 7 \%})$ \\
Entertainment & $109(28.0 \%)$ & $49(27.6 \%)$ & $\mathbf{1 5 8}(\mathbf{2 7 . 9 \% )}$ \\
Social Networking & $99(25.4 \%)$ & $48(27.2 \%)$ & $\mathbf{1 4 7 ( 2 6 . 0 \% )}$ \\
Other & $3(0.5 \%)$ & $2(0.5 \%)$ & $\mathbf{1 ( 0 . 5 \% )}$ \\
\hline
\end{tabular}

familiarity with Boston, $90 \%$ of the sample states having slight or no familiarity with the city. As foreseen, results satisfy the prerequisite that the sample is not familiar with the area where the "scenario" takes place so that participants would use the four web mapping services to perform the tasks in an unknown environment as a means to homogenise the sample further but also to ensure maximum exploration when performing the tasks.

\section{$5 \quad$ Study Results}

Analysis of the collected data was performed per task and service. The analysis was undertaken for: (a) correctness of the participants' responses (effectiveness) and (b) response time (efficiency). Moreover, since all participants evaluated Google Maps, data for this service were analyzed to identify gender differences in performance, if any. Section 5.3 presents the results of such analysis.

All variables have been tested for normality and the majority of the cases, data collected do not follow a normal distribution, thus, all statistical analyses pursued is conducted using non-parametric tests.

\subsection{Task 1: Point Identification}

Within Groups Comparison Regarding the correctness of participants' answers (effectiveness), Tables 3 and 4 depict accuracy of groups in executing Task 1. Table 3 shows that according to the Chi-square tests performed, there is statistically significant moderate correlation between the answers provided by participants of group 2 for Google Maps and MapQuest. The same holds for participants of group 3 for Google Maps and HERE WeGo. 
Table 3. Correlations between answers per pair of services for Task 1.

\begin{tabular}{|l|l|l|l|}
\hline Groups & $\chi^{2}$ & sig. & $r_{\phi}$ \\
\hline 1. Google Maps / Bing Maps & 1.27 & $\mathrm{p}=.260$ & $\mathrm{NA}$ \\
2. Google Maps / MapQuest & 5.45 & $\mathbf{p}=\mathbf{. 0 1 9}$ & $\mathbf{0 . 3 1 2}$ \\
3. Google Maps / HERE WeGo & 5.67 & $\mathbf{p}=\mathbf{0 . 1 7}$ & $\mathbf{0 . 3 1 8}$ \\
\hline
\end{tabular}

Table 4 depicts that, according to the McNemar Tests performed, there is a statistically significant difference between the task's success rate of group 2 participants in Google Maps (better) and MapQuest. On the contrary, for the other two groups no difference can be deduced from the results.

Table 4. Task 1 success rates per group and service.

\begin{tabular}{l|l|l|}
\hline \multicolumn{3}{|c|}{ McNemar Test $\left(\alpha=0.05, \chi^{2}=3.81, d f=1\right)$} \\
\begin{tabular}{|l|l|l|}
\hline Groups & $X^{2}$ & sig. \\
\hline 1. Google Maps (85.45\%) / Bing Maps (87.27\%) & 0.02 & $\mathrm{p}=.880$ \\
2. Google Maps (89.29\%) / MapQuest (73.21\%) & $\mathbf{5 . 5 6}$ & $\mathbf{p}=. \mathbf{0 1 8}$ \\
3. Google Maps (91.07\%) / HERE WeGo (80.36\%) & 3.03 & $\mathrm{p}=.082$ \\
\hline
\end{tabular}
\end{tabular}

Regarding response time and successful completion time (efficiency), Tables 5 and 6 present results of the Wilcoxon matched-pairs signed rank test between response time and successful completion time respectively per pair of services in all three groups. The only statistically significant difference is between Google Maps and Bing Maps for group 1 participants on both cases, where participants seem to be faster on Bing Maps. Finally, there is statistically significant difference of the successful completion time of Task 1 between Google Maps and HERE WeGo, with participants being faster in the latter than the former.

Table 5. Differences between response times for Task 1 in service pairs per group.

Wilcoxon matched-pairs signed-rank test

\begin{tabular}{|l|l|l|l|l}
\hline Groups & N & $\mathrm{z}$ & sig.
\end{tabular}

1. Google Maps (Mdn=220) / Bing Maps (Mdn=98) $55 \mid 3.44$ p=.001

2. Google Maps $(\mathrm{Mdn}=232.5)$ / MapQuest $(\mathrm{Mdn}=253.5) \quad 56|0.60| \mathrm{p}=.546$

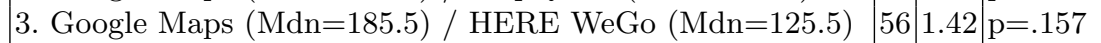

Among Groups Comparison. Comparing among Bing Maps, MapQuest, and HERE WeGo, regarding the correctness of participants' answers (effectiveness), a Chi-square test of independence was performed to examine if there is a relationship between the web mapping service and the ability of the respondents to give a correct answer to the Point Identification task. This relation is non-statistically 
Table 6. Differences between successful completion times for Task 1 in service pairs per group.

Wilcoxon matched-pairs signed-rank test
\begin{tabular}{|l|l|l|l|}
\hline Groups & $\mathrm{N}$ & $\mathrm{z}$ & \multicolumn{1}{l|}{ sig. } \\
\hline 1. Google Maps (Mdn=235) / Bing Maps (Mdn=82.5) & 42 & 3.89 & $\mathbf{p}<\mathbf{. 0 0 1}$ \\
2. Google Maps (Mdn=194) / MapQuest (Mdn=254) & 39 & 1.12 & $\mathrm{p}=.264$ \\
3. Google Maps (Mdn=181) / HERE WeGo (Mdn=110) & 43 & 2.46 & $\mathbf{p = . 0 1 4}$ \\
\hline
\end{tabular}

significant since, $X^{2}(2, N=167)=3.46<\chi^{2}(2,0.05)=5.99, p=.177$. Thus, the service used each time for the execution of the task is not related to the ability of each group to provide a correct answer for the specific task. On the other hand, regarding the successful completion time (efficiency), the Kruskal-Wallis $\mathrm{H}$ test performed shows that the service on which the task was performed has a significant effect on the time of its successful execution $(\mathrm{H}(2)=25.09, \mathrm{p}<.001)$. The Conover post-test shows that the difference between Bing Maps (Mdn = 91.5) and MapQuest $(\mathrm{Mdn}=254)(\mathrm{p}<.001)$ and the one between MapQuest and HERE WeGo $(\mathrm{Mdn}=105)(\mathrm{p}<.001)$ are significant, with participants successfully performing Task 1 on Bing Maps and HERE WeGo faster than those performing it on MapQuest. In contrast, between Bing Maps and HERE WeGo there is no statistically significant difference regarding the successful completion time $(\mathrm{p}=.407)$.

\subsection{Task 2: Locating the Nearest Point of Interest}

Within Groups Comparison. Table 7 shows no statistically significant correlation between answers per service pairs in all three groups for Task 2, according to the Chi-square tests performed. However, regarding success rates, there is statistically significant difference between Google Maps and MapQuest and HERE WeGo for groups 2 and 3 respectively with Google Maps success rates being very low (below 20\%) for both groups (Table 8), according to the McNemar Tests performed. It should be noted that for Task 2 success rates of group 1 are low for both services; Google Maps and Bing Maps.

Table 7. Correlations $r_{s}$ between answers per pair of services for Task 2 .

\begin{tabular}{|l|l|l|l|}
\hline Groups & $\chi^{2}$ & sig. & $r_{\phi}$ \\
\hline 1. Google Maps / Bing Maps & 0 & $\mathrm{p}=.990$ & $\mathrm{NA}$ \\
2. Google Maps / MapQuest & 0.58 & $\mathrm{p}=.445$ & $\mathrm{NA}$ \\
3. Google Maps / HERE WeGo & 1.10 & $\mathrm{p}=.294$ & $\mathrm{NA}$ \\
\hline
\end{tabular}

Regarding response time and successful completion time (efficiency), statistically significant difference can be found only for response time between Google Maps and Bing Maps for group 1 participants; they seem to be faster on Google 
Table 8. Task 2 success rates per group and service.

\begin{tabular}{l}
\hline McNemar Test $\left(\alpha=0.05, \chi^{2}=3.81, d f=1\right)$ \\
\begin{tabular}{|l|l|l|}
\hline Groups & $X^{2}$ & sig. \\
\hline 1. Google Maps (23.64\%) / Bing Maps (30.91\%) & 0.56 & $\mathrm{p}=.456$ \\
2. Google Maps (14.29\%) / MapQuest (50.00\%) & 14.63 & $\mathbf{p}<.001$ \\
3. Google Maps (16.07\%) / HERE WeGo (75.00\%) & 30.18 & $\mathbf{p}<.001$ \\
\hline
\end{tabular}
\end{tabular}

Maps than on Bing Maps (Table 9). Finally, there is no statistically significant difference of the successful completion time of Task 2 in any service pair in any group, partly because no such comparison can be performed for groups 1 and 2 participants since those that successfully executed the task on both services for both groups 2 are very few; 4 and 5 respectively (Table 10).

Table 9. Differences between response times for Task 2 in service pairs per group.

Wilcoxon matched-pairs signed-rank test

\begin{tabular}{|l|l|l|l|l}
\hline Groups & N & Z & sig.
\end{tabular}

\begin{tabular}{|l|l|l|l|l|}
\hline 1. Google Maps (Mdn=242) / Bing Maps (Mdn=402) & 55 & 3.77 & $\mathbf{p}<.001$ \\
\hline
\end{tabular}

2. Google Maps $(\mathrm{Mdn}=231.5) /$ MapQuest $(\mathrm{Mdn}=205) \quad 56 \quad 1.37 \mathrm{p}=.172$

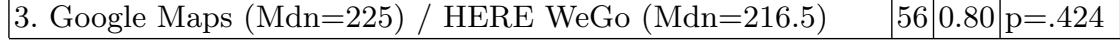

Table 10. Differences between successful completion times for Task 2 in service pairs per group.

\begin{tabular}{l}
\hline Wilcoxon matched-pairs signed-rank test \\
\begin{tabular}{|l|l|l|l|}
\hline Groups & N & s & sig. \\
\hline 1. Google Maps / Bing Maps & 4 & NA & NA \\
2. Google Maps / MapQuest & 5 & NA & NA \\
3. Google Maps (Mdn=199) / HERE WeGo (Mdn=152.5) & 9 & 0.21 & $\mathrm{p}=.834$ \\
\hline
\end{tabular}
\end{tabular}

Among Groups Comparison. Comparing among Bing Maps, MapQuest and HERE WeGo, to assess accuracy, initially, a Chi-square test of independence was performed. This showed statistically significant difference among the three services since, $X^{2}(2, N=167)=21.76<\chi^{2}(2,0.05)=5.99, \mathrm{p}<.001$. Hence, the mapping service used each time to perform Task 2 seems to be related to the ability of each group to find the right answer to the specific task.

Exact Fisher tests showed statistically significant difference between HERE WeGo and the other two (Table 11), with HERE WeGo (42 correct answers; $75 \%$ accuracy) exhibiting the best performance (group 3) followed by MapQuest (28 correct answers; 50\% accuracy, group 2) and lastly by Bing Maps (17 correct 
Table 11. Exact Fisher test results to compare accuracy in performing Task 2.

\begin{tabular}{|l|l|}
\hline Services & Exact Fisher Test (p) \\
\hline Bing Maps / MapQuest & .053 \\
Bing Maps / HERE WeGo & $<.001$ \\
MapQuest / HERE WeGo & $<.011$ \\
\hline
\end{tabular}

answers; $31 \%$ accuracy, group 1). Regarding successful completion time, the Kruskal-Wallis $\mathrm{H}$ test among successful completion times on the three services, shows that the service on which the task was performed has a significant effect on the time of its successful execution $(\mathrm{H}(2)=10.36, \mathrm{p}=.006)$. The Conover posttest shows that the difference between Bing Maps $(\mathrm{Mdn}=368)$ and MapQuest $(\mathrm{Mdn}=201)(\mathrm{p}=.002)$ and between Bing Maps and HERE WeGo (Mdn = 223.5) ( $\mathrm{p}=.018)$ is significant, with participants using MapQuest and HERE WeGo successfully completing the task faster than when using Bing Maps. On the contrary, between MapQuest and HERE WeGo there is no difference in the successful completion time for Task $2(\mathrm{p}=.233)$.

\subsection{Gender Performance}

The entire sample of the study interacted with Google Maps as mentioned in section 3.4. Hence, it was decided to make use of these whole sample data to assess whether there are any differences in performance (effectiveness and efficiency) between genders. In what follows, we present the results of the comparison between male and female participants in both tasks regarding effectiveness and efficiency.

Initially, a Chi-square test of homogeneity was performed to examine the homogeneity of the three groups in terms of the correctness of the answers to both tasks on Google Maps. Results indicate homogeneity of the three groups for both tasks (Task 1: $X^{2}(2, N=167)=0.90<\chi^{2}(2,0.05)=5.99, \mathrm{p}=.636$, and Task 2: $\left.X^{2}(2, N=167)=1.85<\chi^{2}(2,0.05)=5.99, \mathrm{p}=.396\right)$. Therefore, results of the three groups indicate that they come from the same population and they can be used as a whole to examine how male and female participants interacted with Google Maps.

Task 1: Point Identification. Before proceeding with comparing performance between genders, correlations between answers and time taken to respond have been calculated.

Table 12 shows point-biserial correlations between answers and response time of male and female participants for Task 1. For male participants as well as for female ones, the correlation between answers and time taken to perform the task is almost zero and non-statistically significant. This practically means that answers' accuracy of both groups (males and females) was not affected by the time they took to perform the task. As proved by the results (Section 5.1), Task 1 is quite easy, thus participants could perform the task correctly if we 
take into consideration the success rates independently of the time they took to complete the task, hence the zero correlation. Regarding the correctness of

Table 12. Point-biserial correlation between answers and response time for Task 1 for male and female participants

\begin{tabular}{|l|l|l|l|}
\hline Participants & $\mathbf{N}$ & $r_{p b}$ & sig. \\
\hline Males & 113 & -0.145 & $\mathrm{p}=.125$ \\
Females & 54 & -0.196 & $\mathrm{p}=.163$ \\
\hline
\end{tabular}

participants' answers (effectiveness), results of the Chi-square text indicate a non-significant difference in the correctness of $91 \%$ (103 out of 113) of male participants, compared to the $83 \%$ (45 out of 54) correctness of female participants $\left.X^{2}(1, N=167)=2.21<\chi^{2}(1,0.05)=3.84, \mathrm{p}=.137\right)$. While, regarding the response time and successful completion time (efficiency) the Mann-Whitney test performed, leads to the conclusion that male participants $(\mathrm{Mdn}=190)$ were statistically significantly faster in their response time than females $(\mathrm{Mdn}=264.5)$ $(\mathrm{U}=2475.5, \mathrm{p}=.025)$. The same holds when comparing between successful completion time; male participants $(\mathrm{Mdn}=184)$ who performed the task correctly, did it faster than female participants $(\mathrm{Mdn}=264)(\mathrm{U}=1873.5, \mathrm{p}=.032)$.

Task 2: Locating the Nearest Point of Interest. Again, as an initial step, correlations between answers and time taken to respond have been calculated.

Table 13 shows point-biserial correlations between answers and response time of male and female participants for Task 2. For all participants regardless of gender, the correlation between answers and time taken to perform the task is almost zero and non-statistically significant, as similar as for Task 1. This practically means that accuracy of both groups (males and females) was not affected by the time they took to perform the task. As proved by the results (see Section 5.2), Task 2 was not successful, meaning that participants, even those who took more time to complete the task, could not improve their effectiveness, hence the zero correlation.

Table 13. Point-biserial correlation between answers and response time for Task 2 for male and female participants

\begin{tabular}{|l|l|l|l|}
\hline Participants & $\mathbf{N}$ & $r_{p b}$ & sig. \\
\hline Males & 113 & 0.026 & $\mathrm{p}=.783$ \\
Females & 54 & 0.084 & $\mathrm{p}=.547$ \\
\hline
\end{tabular}

As for the correctness of participants' answers (effectiveness), results of the Chi-square test indicate a non-significant difference in the correctness of 19\% (22 out of 113) of male participants, compared to the $15 \%$ ( 8 out of 54 ) correctness 
of female participants $\left.X^{2}(1, N=167)=0.54<\chi^{2}(1,0.05)=3.84, \mathrm{p}=.524\right)$. The same holds, for both response and successful completion times (efficiency), since according to the Mann-Whitney test, there is no statistically significant difference between male $(\mathrm{Mdn}=228)$ and female $(\mathrm{Mdn}=239)$ participants response time $(\mathrm{U}=2707, \mathrm{p}=.240)$. The same holds when comparing between successful completion time; there is no difference between successful completion time of male $(\mathrm{Mdn}=189)$ and female $(\mathrm{Mdn}=314)$ participants $(\mathrm{U}=52.5$, $\mathrm{p}=.101)$.

\section{Overall Discussion and Conclusions}

Study results show that although digital natives are familiar with the use of computers and smart devices, they have difficulties when exploring functionalities of web mapping services. This is indicated by the success rates in the two tasks they had to perform.

Specifically:

- Task 1 seems to be the one that facilitated the participants since for all services success rates are between $73 \%$ - 91\%. For group 2 participants though, there is a statistically significant difference in their success rates; participants performed better on Google Maps that on MapQuest. Moreover, considering genders' performance in interacting with Google Maps, no difference is indicated through the survey's sample for Task 1; the task seems to be equally easy to perform successfully by both males and females.

- Success rates for Task 2 are disappointing since in two services (Google Maps and Bing Maps) they are below 31\%. However, MapQuest and HERE WeGo present higher success rates $(50 \%$ and $75 \%$ respectively) than the other two showing that for this task, the service used to perform the task was significant for achieving successful results as also proven by the Chi-square test of independence. On Google Maps, both male and female participants reached similar success rates indicating no difference in effectiveness related to gender.

Regarding response time and time of successful task completion, the following are observed:

- In Task 1, response times and times of successful task completion, were shorter on Bing Maps and on HERE WeGo than those on Google Maps (statistically significant differences calculated, see Tables 5 and 6 ) but that does not hold for MapQuest. This result is partly consistent with the trialby trial sequential effect [8] which suggests that survey respondents who are required to use a series of tools/or applications, they tend to perform faster each time they perform the tasks they are called to, since they become familiarized with the process the following times. However, if we consider the results for Task 2, this is also partly supported but does not constitute a statistically significant difference in favor of the second map service used by each group (see Tables 9 and 10). 
- Numerous studies have shown that effectiveness tends to increase especially when there is no time pressure, as it is in this case, where participants had no time limit imposed to them for completing a task. However, the accuracy curve does not always seem to increase with response time [9]. Something similar seems to be happening in the present study as indicated by the almost zero correlation between the answers' correctness of both male and female participants and the time they took to perform the tasks on Google Maps.

- Finally, regarding to what is referred to as "rapid guessing" [10] observed due to lack of motivation or interest by the survey participants, after analysing the fifth percentile of response times, we can say that "rapid guesses" are correct for Task 1 in all four services, while mostly wrong for Task 2. This highlights once again the low degree of difficulty that Task 1 presented to participants. On the opposite, the degree of difficulty of Task 2 affected quick responses correctness to such an extent that they can be characterized as "rapid guesses" on the part of the participants.

Gender performance results do not indicate any significant difference between male and female participants. Both groups are similar in accuracy in both tasks performed. A slight difference in speed favoring male participants in Task 1 alone is inconclusive for stating whether there is a systematic faster performance of males versus females, especially since no such difference exists for the "difficult" Task (Task 2).

As an overall conclusion, this study highlighted that significant part of participants' performance and interaction with web mapping services are mostly task-driven than service- or gender- driven; a difficult task downplays any difference among services usability issues and between genders and it seems to be the overarching factor of participants' performance.

\section{Acknowledgements}

Authors would like to thank all students who participated to the user study.

\section{References}

1. Prensky, M.: Digital natives, digital immigrants part 1. On the Horizon, 9(5), 3-6 (2001). https://doi.org/10.1108/10748120110424816

2. Brooke, J.: SUS: a retrospective. Journal of Usability Studies, 8(2), 29-40 (2013).

3. Bevan, N., Carter, J., Harker, S.: ISO 9241-11 Revised: What Have We Learnt About Usability Since 1998?. In: Kurosu, M. (ed) Human-Computer Interaction: Design and Evaluation. HCI 2015. LNCS, vol. 9169, Springer, Cham (2015). https://doi.org/10.1007/978-3-319-20901-2_13

4. Nivala, A.-M., Brewster, S., Sarjakoski, T.L.: Usability Evaluation of Web Mapping Sites. The Cartographic Journal, 45(2), 129-138 (2008). https://doi.org/10.1179/174327708X305120 
5. Wachowicz, M., Cui, L., Vullings, W., Bulens, J.: The effects of web mapping applications on user satisfaction: an empirical study. In: Peterson, M.P. (ed) International Perspectives on Maps and the Internet. Lecture Notes in Geoinformation and Cartography. Springer, Berlin, Heidelberg (2008). https://doi.org/10.1007/9783-540-72029-4_25

6. Wang, C.: Usability evaluation of public web mapping sites. The International Archives of the Photogrammetry, Remote Sensing and Spatial Information Sciences, 60(4), 285-289 (2014). https://doi.org/10.1179/174327708X305120

7. Panko, R.: The Popularity of Google Maps: Trends in Navigation Apps in 2018. The Manifest (2018). https://themanifest.com/mobile-apps/popularitygoogle-maps-trends-navigation-apps-2018. Last accessed 03 February 2021

8. Baayen, H., Milin, P. R.: Analyzing reaction times. International Journal of Psychological Research, 3(2), 12-28 (2010). https://doi.org/10.21500/20112084.807

9. De Boeck, P., Jeon, M.: An Overview of Models for Response Times and Processes in Cognitive Tests. Frontiers in Psychology, 10(102), (2019). https://doi.org/10.3389/fpsyg.2019.00102

10. Ratcliff, R.: Methods for dealing with reaction time outliers. Psychological Bulletin, 114, 510-532 (1993). 